\title{
Editorial
}

\section{Combating Kinetoplastid diseases}

\section{Alberto MR Dávila*1 and Kevin M Tyler*2}

\author{
Address: ${ }^{1}$ Departamento de Bio química e Biologia Molecular, Instituto Oswaldo Cruz, Fiocruz. Av. Brasil 4365, Rio de Janeiro, CEP 21045-900, \\ Brasil and ${ }^{2}$ Dept of Pathology, University of Northwestern Medical School, Ward 6-140, 303 E. Chicago Ave. Chicago 60611, USA \\ E-mail: Alberto MR Dávila* - davila@ioc.fiocruz.br; Kevin M Tyler* - k-tyler@northwestern.edu \\ *Corresponding authors
}

Published: 5 July 2002

Kinetoplastid Biology and Disease 2002, I:6

This article is available from: http://www.kinetoplastids.com/content/l/I/6

(c) 2002 Dávila and Tyler; licensee BioMed Central Ltd. Verbatim copying and redistribution of this article are permitted in any medium for any purpose, provided this notice is preserved along with the article's original URL.

\section{Ancient Plagues, Modern Epidemics}

Today's Kinetoplastida form a diverse order of flagellated protozoans that have evolved from an ancient lineage, rooted near the base of the eukaryotic tree. The disease caused by some species of the Order Kinetoplastida have always plagued mankind, and today most are at least as prevalent as they have ever been. Kinetoplastid parasites cause disease in humans, animals and plants, severely affecting human health and retarding agriculture development in less developed countries. Sleeping sickness (caused by pathogenic subspecies of Trypanosoma brucei), Chagas disease (caused by Trypanosoma cruzi) and the Leishmaniases (caused by Leishmania $s p p$ ) are the major human diseases caused by kinetoplastids. According to the World Health Organization "sleeping sickness" affects more than 60 million men, women and children in 36 countries of sub-Saharan Africa, most of which are among the least developed countries in the world. In many of these countries sleeping sickness is currently epidemic, re-emerging in some as a greater cause of morbidity than even HIV/AIDS. T. cruzi currently infects 14 million people in Latin America. It is the agent of Chagas disease, the leading infectious cardiomyopathy in the world. The leishmaniases and the suffering they cause threaten 350 million women, men and children in 88 countries around the world, 72 of which are developing countries. In addition to their medical importance kinetoplastid parasites also cost developing nations millions of dollars in lost agricultural revenues, since other kineto-
Received: 16 June 2002

Accepted: 5 July 2002 plastids are pestilences that strike agricultural produce from crops, to fish to cattle.

\section{A Problem with Communication?}

The study of kinetoplastid disease is now over a hundred years old and has always graced the forefront of medical research. A casual search of PubMed, which lists most articles published in major periodic journals over the past 35 years, shows over 25,000 articles published on kinetoplastids. This is a number comparable to important infectious diseases in the Western Hemisphere such as influenza, herpes or measles. Research has not, however, translated into therapeutics, prophylactics or even cheap effective diagnostics. It is widely accepted that the majority of research on these organisms has been driven by expertise that is out of step with field needs. Researchers on kinetoplastids have remained resilient to communication with health systems researchers who take a macroscale view of how disease control options might be integrated into declining and impoverished public health and animal health systems. Conversely, it has been easy for the therapeutic potential of advances made through research to fail to progress all the way to the field, since news carried in traditional, subscription based scientific journals is inaccessible to most workers in developing nations. Even amongst western researchers working on these diseases communication is poor. Those working on human disease publish in medical journals, whilst those working on the organisms themselves publish in basic science journals, those in veterinary disease publish in distinct 
veterinary journals and those in epidemiology and public health use their own sets of journals. The result is that each discipline is largely ignorant of advances made by other parts of the field, progress is impeded, and essentially similar studies are often reproduced multiple times with varying quality. Finally, there is no global advocate and no centralized resource for this group of diseases: This has effectively ceded to HIV, TB, malaria and hemorrhagic fevers the global agenda and vast majority of the world's public health funding. This has meant that this group of diseases is seriously under-represented compared with the grief and suffering that they cause and has left a legacy of a lethal and incurable disease like sleeping sickness unchecked in much of Africa.

\section{What are our objectives?}

The case is far from hopeless, pragmatic and cost effective programs such as "The Southern Cone Initiative" (which has eliminated transmission of T. cruzi from much of South America) demonstrate how public health strategies can impact on such disease, when political will can be harnessed. Our goal is first and foremost to promote effective communication about this group of diseases using the Internet. We hope to fundamentally change the way in which scientists and clinicians, veterinarians and public health workers interact and relate to one another by providing a forum to broadly educate everyone in the field. Our secondary goal is to provide as central resource to act as a reservoir of information and a protagonist for encouraging the development of resources directed at combating this group of diseases. In sum, to provide a free, comprehensive and accessible resource to anyone interested in learning more about this group of diseases. To achieve these objectives we aim to establish an Internet portal [http://www.trypanosome.com/]. This will take the form of a centralized resource featuring its own online journal, Kinetoplastid Biology and Disease (KBD), as well as links to other key online resources pertaining to kinetoplastid disease:

$>$ Databases (contact directory of professionals working on these diseases world-wide, images bank for educational and promotional materials)

Conferences (yearly organization, archives of previous conferences)

> On-line discussions (Tryplink, Newsgroup and archives, live discussion forum)

Infections and diseases (description of trypanosomatid parasites, the diseases they cause and their control)
- Genomes (links to kinetoplastid specific genome and proteome databases, genomics and proteomics resources.)

$>$ Links (to books, jobs and related sites)

All the information in the centralized site will be freely available worldwide to anyone with a networked computer; allowing bench and field researchers rapid communications with which to describe disease trends, outbreaks, and to recommend control measures. Hopefully, this will also serve to improve links and reduce distances between people in the less developed, affected countries and those in developed ones.

\section{Kinetoplastid Biology and Disease [http://kineto- plastids.com]}

The advent of KBD enables the free dissemination of scientific information on diseases and control to anyone in the world with a networked computer. This is critical, since most scientific journals are not free and even the ones with cheaper subscription rates may not be accessible to researchers, clinicians, and field researchers in the poorest and affected developing countries. Moreover, the journal will serve as a focus in which the whole kinetoplastid community can participate: to educate, notify and debate about progress and direction. We hope our new journal will serve as a vehicle to promote pragmatic research and as a practical first step in tackling some of the communication difficulties that face those concerned with the eradication of these diseases.

KBD is created as peer-review journal [http://kinetoplastids.com/info/about/], freely available to anyone in the world with a networked computer. We boast a word-class editorial board [http://kinetoplastids.com/edboard/] and a unique focus "kinetoplastids" - their biology, their vectors and the diseases they cause.

\section{Acknowledgements}

We would like to thank the editorial board for their support in this endeavour; in particular, those who submitted papers to past Internet conferences and those who have contributed to this first issue. Specifically, we would like to thank Hooman Momen for his initial and continuous support, Ami Ganguli for his ongoing computer support and Marli L. Costa for her editorial assistance. We would also like to thank David Molyneux for his assistance in the writing of this forward. We also thank the "Vice-Presidência de Desenvolvimento Institucional, Informação e Comunicação" and "Instituto Oswaldo Cruz, Fiocruz" for the support. 